\title{
On differentiable compactifications of the hyperbolic space
}

\author{
Benoît Kloeckner
}

October 24, 2018

\begin{abstract}
The group of direct isometries of the hyperbolic space $\mathbb{H}^{n}$ is $G=$ $\mathrm{SO}_{0}(\mathrm{n}, 1)$. This isometric action admits many differentiable compactifications into an action on the closed $n$-dimensional ball. We prove that all such compactifications are topologically conjugate but not necessarily differentiably conjugate. We give the classifications of real analytic and smooth compactifications.
\end{abstract}

\section{Introduction}

\subsection{Goal}

The group $G=\mathrm{SO}_{0}(\mathrm{n}, 1)$ acts on the $n$-dimensional open ball as the isometries for the hyperbolic riemanniann metric (we denote this action by isom). We study the differentiable compactifications of this action into an action of $G$ on the closed ball $\overline{\mathbb{B}}^{n}$, that is to say the differentiable actions of $G$ on the closed ball such that the restriction to the open ball is differentiably conjugate to isom.

We concentrate on two levels of regularity: "differentiable" shall always mean smooth or real analytic.

One analytic compactification is given by the continuous extension of the action of $G$ in the Klein model of the hyperbolic space. We shall give later on more details about this compactification, called "projective" and denoted by proj.

It appears that up to an analytic change of coordinates, there is a countable familly of analytic compactifications. The classification is more complicated in the smooth case, but we get a simple description of all smooth compactifications.

To describe the compactifications, we use half-space charts of $\overline{\mathbb{B}}^{n}$ missing only one point.

Theorem 1 In some half space chart the conjugation of proj by a change of coordinates of the form:

$$
\varphi_{p}:\left(x_{1}, \ldots, x_{n-1}, y\right) \longmapsto\left(x_{1}, \ldots, x_{n-1}, y^{p}\right)
$$

(where $p$ is a positive integer) is well defined in the open ball and continuously extendable into an analytic action on the closed ball. Thus for each positive integer $p$ we get an analytic compactification of isom. Moreover, any analytic 
compactification is analytically conjugate to one of these and no two different $p$ 's give conjugate compactifications.

We shall prove this result when $n \geqslant 3$; the case $n=2$ can be deduced from works of Schneider [3] and Stowe [4] exposed by Mitsumatsu 2].

Theorem 2 In some half space chart the conjugation of proj by a change of coordinates of the form:

$$
\varphi_{f}:\left(x_{1}, \ldots, x_{n-1}, y\right) \longmapsto\left(x_{1}, \ldots, x_{n-1}, f(y)\right)
$$

(where $f: \mathbb{R}^{+} \longrightarrow \mathbb{R}^{+}$is a homeomorphism that is smooth and a diffeomorphism of $\mathbb{R}^{+*}$ ) is well defined in the open ball and is continuously extendable into a smooth action on the closed ball if and only if

$$
f / f^{\prime} \text { is smooth. }
$$

Thus for each map $f$ satisfying (3) we get a smooth compactification of isom. Moreover, if $n \geqslant 3$ any smooth compactification is analytically conjugate to one of these. Two different $f$ 's give conjugate compactifications if and only if they are smoothly conjugate.

Condition (3) is automatically satisfied if $f$ is non-flat (i.e. has some nonzero derivative in 0 ).

\section{Remarks}

1. By " $f$ is smooth" we mean that it can be prolonged into a smooth map defined on $]-\varepsilon,+\infty$ [ where $\varepsilon$ is some positive real number. Equivalently, $f$ is smooth on $\mathbb{R}^{+}$if and only if all its derivatives converge in 0 ,

2. some flat maps satisfy condition (31) while others do not. For example, if $f_{1}=x \longmapsto \exp \left(-x^{-2}\right)$ and $f_{2}=x \longmapsto \exp \left(-x^{-\frac{3}{2}}\right)$ we have $\left(f_{1} / f_{1}^{\prime}\right)(x)=$ $\frac{1}{2} x^{3}$ but $\left(f_{2} / f_{2}^{\prime}\right)(x)=\frac{2}{3} x^{\frac{5}{2}}$,

3. in general, $f$ is singular at 0 . Otherwise, the compactification given by $\varphi_{f}$ is conjugate to proj,

4. in the two-dimensional case, it appears [1] that there are only two smooth compactifications of isom (namely the projective one and the conformal one, see 0.2 .1 for a definition) that are algebraic, i.e. given by a linear representation of $G$ after projectivization and restriction to an embedded manifold that is a union of orbits. Thanks to Theorem [2] it is easy to generalize this result to higher dimensions (an algebraic action of $\mathrm{SO}_{0}(1, \mathrm{n})$ on the closed $n$-ball gives by restriction to a totally geodesic plane an algebraic action of $\mathrm{SL}_{2}(\mathbb{R})$ on the closed 2-ball). 

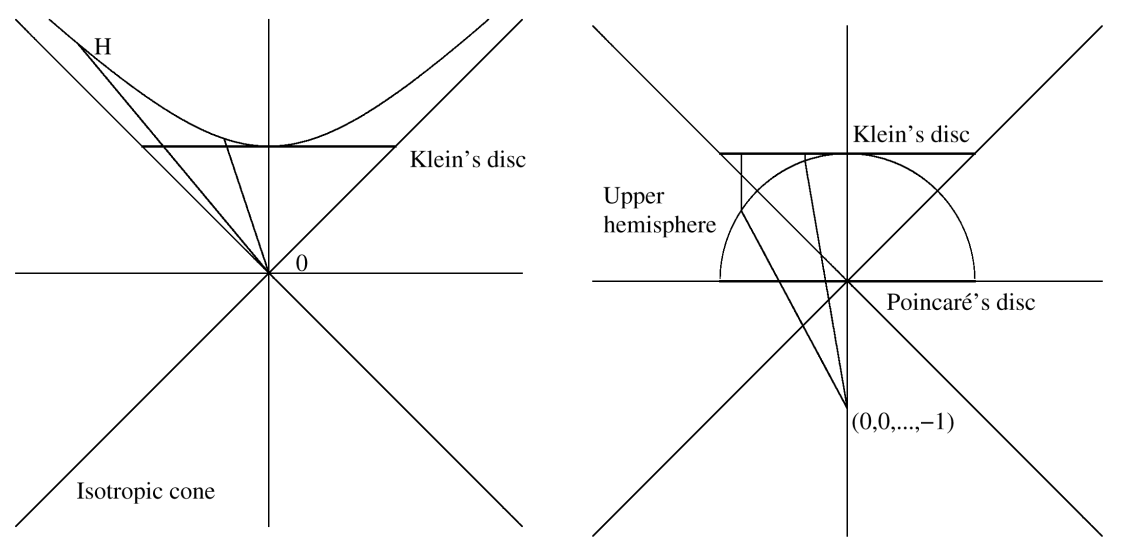

Figure 1: Construction of the Klein ball and Poincaré ball models

\subsection{Recalls and notations}

\subsubsection{The projective and conformal compactifications}

Two models of the isometric action of $\mathrm{SO}_{0}(\mathrm{n}, 1)$ on the hyperbolic space are well-known: the Poincaré ball and the Klein ball.

Let us recall the construction of these models. Put on $\mathbb{R}^{n+1}$ a Lorentzian quadratic form $Q=x_{1}^{2}+x_{2}^{2}+\cdots+x_{n}^{2}-y^{2}$ and consider the hypersurface $H$ defined by $Q=-1$ and $y>0$. $Q$ induces a scalar product on each tangent space of $H$, thus defines a riemannian structure: the hyperbolic space $\mathbb{H}^{n}$, on which $\mathrm{SO}_{0}(\mathrm{n}, 1)$ acts naturally by isometries. A central projection of center 0 of $H$ on the disc of radius 1 of the hyperpane $y=1$ gives the Klein ball model. If we project vertically the Klein ball on the upper half-sphere of radius 1 of $\mathbb{R}^{n+1}$ and then project the half-sphere stereographically from the point $(0,0, \ldots, 0,-1)$ on the plane $y=0$, we get the Poincaré ball model (see figure 1).

In both of these models, the isometric action of $\mathrm{SO}_{0}(\mathrm{n}, 1)$ appears to admit continuous extensions to the closed ball into an analytic action.

The extension in the Poincare model is called the conformal action: it preserves the conformal euclidian structure of the closed unit ball. We denote this action by conf, and the action of an element $g \in G$ by conf $g$.

The extension in the Klein model is called the projective action: it preserves the projective structure of the closed ball, subset of $\mathbb{P}\left(\mathbb{R}^{n+1}\right)$ (in particular, the projective action maps straight lines into straight lines). We denote this action by proj, and the action of an element $g \in G$ by $\operatorname{proj}_{g}$. We also denote by $\operatorname{proj}_{X}$ the vector field given by the projective action of an element $X$ of the Lie algebra $\mathfrak{g}$ of $G$.

It is easy to see that the conformal and projective actions are topologically conjugate, but they are not differentiably conjugate.

Let us give a purely geometrical proof.

In the conformal model, two asymptotic geodesics are always tangent but in the projective model, for any direction in the tangent space of a point of the boundary there is exactly one geodesic tangent to it (we shall see that this property of proj plays a fundamental role in Theorems 10 and 2). 
Therefore, the group of the parabolic elements of $G$ which stabilize a given point of the boundary has a common proper direction transversal to the boundary (in particular their linear parts are simultaneously diagonalisable) for the conformal action but not for the projective one, hence these two compactifications cannot be differentiably conjugate.

\subsubsection{Half-space charts}

We denote by $\mathbb{R}^{n+}$ the open $n$-dimensional half space and by $\overline{\mathbb{R}}^{n+}$ its closure in $\mathbb{R}^{n}$. We shall use the canonical coordinates system $\left(x_{1}, x_{2}, \ldots, x_{n-1}, y\right)$, therefore $\mathbb{R}^{n+}=\left\{\left(x_{1}, \ldots, x_{n-1}, y\right) \in \mathbb{R}^{n} ; y>0\right\}$.

The extension to the boundary of the isometric action of the Poincaré halfspace is the conformal action written in some half-space chart of the closed ball. We denote this chart by $\mathcal{P C}$, it is given by: a central projection of the Poincaré disc from the south pole $(0,0, \ldots, 0,-1)$ on the upper half-sphere, composed with a stereographic projection of center $(0,0, \ldots, 0,1,0)$ on the vertical $n$ dimensional half-space defined by $x_{n}=0$ (identified with $\mathbb{R}^{n+}$ ).

If we compose the vertical projection of the Klein Ball on the upper halfsphere with the stereographic projection of center $(0,0, \ldots, 0,1,0)$ on the vertical $n$-dimensional half-space defined by $x_{n}=0$ and then with the following map:

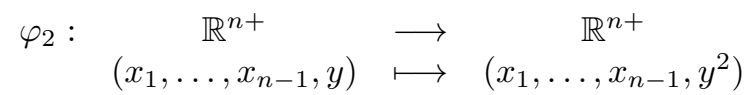

it appears that we get a chart, denoted by $\mathcal{K C}$. An explicit change of coordinates defining this chart from the projective coordinates is given by:

$$
\left[1: x_{1}: x_{2}: \cdots: x_{n-1}: y\right] \longmapsto\left(\frac{x_{1}}{1-y}, \frac{x_{2}}{1-y}, \ldots, \frac{1-\sum_{i=1}^{n-1} x_{i}^{2}-y^{2}}{(1-y)^{2}}\right)
$$

Hence conf corresponds in Theorem 1 to the case $p=2$. In particular conf and proj are Hölder- $\frac{1}{2}$ conjugate.

\section{Topological uniqueness}

We saw that the conformal and projective actions are topologically conjugate. More generally we prove the following fact:

Proposition 3 There is up to topological conjugacy only one continuous action of $G$ on the closed ball such that the action on the interior is homeomorphic to isom.

This uniqueness enables us to use the following definition:

Definition 4 Throughout this paper, by a $\mathcal{C}^{k}$ compactification ( $k$ is $\infty$ or $\omega$ ) of isom we shall mean a homeomorphism $\varphi$ of the closed ball which is a $\mathcal{C}^{k}$ diffeomorphism in the interior and such that for each $g \in G, \varphi^{-1} \circ \operatorname{proj}_{g} \circ \varphi$ (defined on the open ball) admits a $\mathcal{C}^{k}$ extension to the closed ball. 


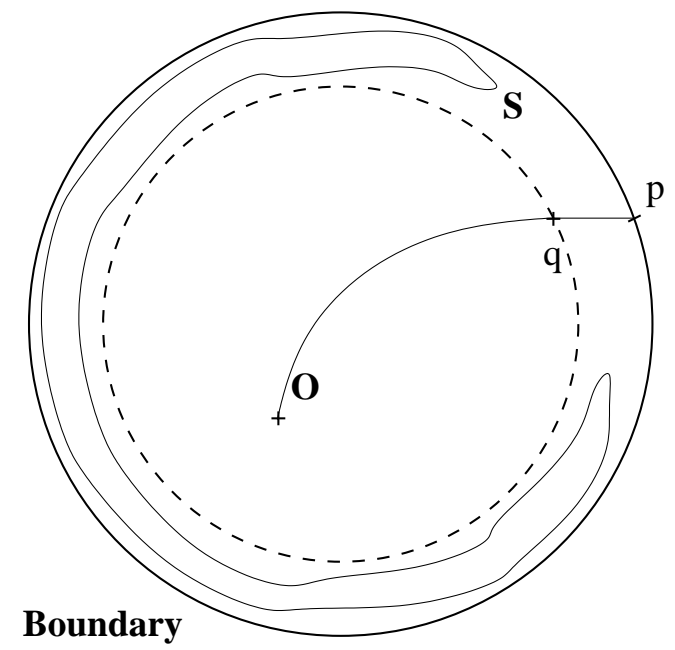

Figure 2: If $S$ does not have $O$ in its interior there may exist a point $p$ in the boundary not close to $S$

In other words, we do not distinguish the action from the map which topologically conjugates it to proj.

The choice of the projective action as a "reference" compactification comes from the classification results (Theorems 1 and 2).

Let us prove Proposition 3

\subsection{First step : action on the boundary}

We shall first prove that the restriction of $\rho$ to the boundary sphere is topologically conjugate to that of proj.

Since the Hadamard boundary of the Poincaré ball is the only $(n-1)$ spherical homogeneous space of $G$ (up to topological conjugacy), it is sufficient to prove that $\rho$ is transitive on the boundary.

Since $\rho$ is homeomorphic to isom in the open ball, the orbits in the open ball under the action $\rho(\mathrm{SO}(\mathrm{n})$ ) (where $\mathrm{SO}(\mathrm{n})$ is seen as a subgroup of $G$ ) are: a fixed point $O$, and topological spheres disconnecting the open ball in two connected components. Moreover, $O$ is in the interior component of all these spherical orbits.

Take a point $x$ on the boundary of the closed ball and choose a real number $\varepsilon>0$, smaller than the distance between the boundary and $O$.

By uniform continuity ( $\mathrm{SO}(\mathrm{n})$ is compact), there is an orbit $S$ of $\rho(\mathrm{SO}(\mathrm{n}))$ and a point $x^{\prime} \in S$ such that for all $g \in \mathrm{SO}(\mathrm{n}), \rho(g) \cdot x$ is $\varepsilon$-close to $\rho(g) \cdot x^{\prime} \in S$. Therefore $S$ is in the neigborhood of size $\varepsilon$ around the boundary. Since its interior contain $O$ (this condition is important, figure 2 shows a counterexample), any point of the boundary is $\varepsilon$-close to $S$. Indeed, if $p$ is in the boundary and not $\varepsilon$-close to $S$, there is a path avoiding $S$ and connecting $p$ to a point $q$ not $\varepsilon$-close to the boundary. Since $S$ is in an $\varepsilon$-neighborhood of the boundary, there is a path avoiding $S$ and connecting $q$ to $O$, thus there is a path avoiding $S$ and connecting $p$ (which is outside $S$ ) to $O$ (which is inside $S$ ), a contradiction. 
The orbit of $x$ under $\rho(\mathrm{SO}(\mathrm{n}))$ is $\varepsilon$-dense in the boundary for all sufficiently small $\varepsilon$, thus it is dense. It is also compact, thus $\rho$ must be transitive on the boundary.

\subsection{Second step : closure of a geodesic}

Let $\psi$ be the homeomorphism of the open ball which conjugates isom (written in the Klein model) and the restriction of $\rho$ to the open ball.

Let $L$ be a projective geodesic. We shall prove that the closure of $\psi(L)$ in the closed ball has exactly two points in the boundary.

We denote by $\operatorname{EP}(L)$ the intersection of the boundary and of the closure of $\psi(L)$.

Since $\psi(L)$ is non compact and closed in the open ball, $\operatorname{EP}(L)$ is non empty. It is also compact and globally invariant under the action of $\rho(g)$ for all $g$ whose projective action leaves $L$ globally invariant. The projective action of these $g$ 's has two orbits in the boundary : the first contains the two endpoints of $L$ and the second all the other points of the boundary. Thus, since the restriction of $\rho$ to the boundary is topologically conjugate to that of $\operatorname{proj}, \operatorname{EP}(L)$ has two points or is the entire boundary (it is compact).

If $\operatorname{EP}(L)$ were the entire boundary, let $x$ be a point in the boundary not fixed by some rotation $\rho(g)$ which leaves $\psi(L)$ pointwise invariant. Let $\left(x_{n}\right)$ be a sequence of points of $\psi(L)$ such that $\lim x_{n}=x$. Then $\rho(g) \cdot x_{n}=x_{n}$ thus by continuity $\rho(g) \cdot x=x$, a contradiction.

Therefore $\psi(L)$ has exactly two endpoints (one for each half of the geodesic). Moreover, the endpoints are determinated by their stabilizer, thus the images of two asymptotic half-geodesics have the same endpoint and any point of the boundary is the endpoint of some geodesic.

\subsection{Third step : extension of $\psi$}

We can extend $\psi$ into a one-to-one map (denoted by $\widetilde{\psi}$ ) of the closed ball into itself : $\widetilde{\psi}$ maps the endpoint of a half geodesic to the endpoint of its image by $\psi$. Then $\widetilde{\psi}$ conjugates $\rho$ and proj.

We shall prove that $\widetilde{\psi}$ is a homeomorphism. Since it is one-to-one and the closed ball is compact, it is sufficient to prove its continuity. Since it coincides with $\psi$ in the interior, it is sufficient to prove its continuity at all points of the boundary.

Let $x$ be a point of the boundary and $\left(x_{n}\right)_{n \in \mathbb{N}}$ be a sequence of points of the closed ball such that $\lim x_{n}=x$.

Let $L$ be the closure of a half-geodesic of endpoint $x$. Let $o$ be a point of $L, L_{n}$ be the half-geodesic with endpoint $o$ and containing $x_{n}, P_{n}$ be the totally geodesic plane containing both $L$ and $L_{n}$ and $Q_{n}$ be the maximal totally geodesic subspace orthogonal to $P_{n}$. There is a sequence $\left(g_{n}\right)_{n \in \mathbb{N}}$ of elliptic elements of $G$ such that $\operatorname{proj}_{g_{n}}$ is identity on $Q_{n}, \operatorname{proj}_{g_{n}}$ globally stabilizes $P_{n}$ and $\operatorname{proj}_{g_{n}} \cdot(L)=L_{n}$ for all $n$. Let $Y_{n}=\operatorname{proj}_{g_{n}^{-1}} \cdot x_{n}$. For all $n, y_{n} \in L$ and since $x_{n}$ has $x$ for limit, the sequence of angles of the rotations $g_{n}$ has limit zero and $\lim _{n \rightarrow \infty} g_{n}=1$. 
Then

$$
\begin{aligned}
\widetilde{\psi}\left(x_{n}\right) & =\widetilde{\psi}\left(\operatorname{proj}_{g_{n}} \cdot y_{n}\right) \\
& =\rho\left(g_{n}\right) \cdot \widetilde{\psi}\left(y_{n}\right)
\end{aligned}
$$

and since $y_{n}$ has for limit the endpoint of $L$, by definition $\widetilde{\psi}\left(y_{n}\right)$ has for limit the endpoint of $\widetilde{\psi}(L)$, that is to say $\widetilde{\psi}(x)$. Thus the uniform continuity of $\rho$ in some neighborhood of $1 \in G$ ensures $\lim _{n \rightarrow \infty} \widetilde{\psi}\left(x_{n}\right)=\widetilde{\psi}(x)$ and $\widetilde{\psi}$ is continuous. The proof is complete.

\section{Proof of Theorems 1 and 2}

\subsection{Main part}

We shall start with a lemma which contains the heart of the proof. Recall that $k$ is always $\infty$ or $\omega$.

Lemma 5 Let $\varphi$ be a $\mathcal{C}^{k}$ compactification of the isometric action of $G$ such that the inverse images of the projective geodesics are border-transversal $\mathcal{C}^{k}$ submanifolds of the closed ball. Then, up to a $\mathcal{C}^{k}$ change of coordinates, $\varphi$ can be written in half-space charts (the chart at the goal being $\mathcal{K C}$ ) in the following form:

$$
\varphi=\left(x_{1}, \ldots, x_{n-1}, y\right) \longmapsto\left(x_{1}, \ldots, x_{n-1}, f(y)\right)
$$

where $f$ is a $\mathcal{C}^{k}$ map.

We recall that for a map $f$ defined on $\mathbb{R}^{+}$, being $\mathcal{C}^{k}$ means that $f$ is $\mathcal{C}^{k}$ on $\mathbb{R}^{+*}$ and can be prolonged in a neighborhood of 0 into a $\mathcal{C}^{k}$ map.

Proof: in the Lie algebra $\mathfrak{g}$ of $G$ we shall denote by $X_{1}, X_{2}, \ldots, X_{n-1}$ a basis of the vector space of the parabolic transformations which fix $\infty$ (the only point missed by the half-space chart). The corresponding vector fields for the projective action are denoted by $\operatorname{proj}_{X_{1}}, \ldots, \operatorname{proj}_{X_{n-1}}$. By definition, in the chart $\mathcal{K C}$ they may be written as $\operatorname{proj}_{X_{i}}=\frac{\partial}{\partial x_{i}}$.

Without loss of generality, we can suppose that $\varphi(0)=0, \varphi(\infty)=\infty$ and, choosing wisely the $\overline{\mathbb{R}}^{n+}$ chart for the source of $\varphi$, that $\varphi^{*}\left(\operatorname{proj}_{X_{i}}\right)=\frac{\partial}{\partial x_{i}}=$ $\operatorname{proj}_{X_{i}}$ for each $i$ (the subgroup generated by $\varphi^{*}\left(\operatorname{proj}_{X_{i}}\right)$ 's acts freely and is abelian.)

Note that in the chart $\mathcal{K C}$ any vertical line is a geodesic.

We shall first prove that via a differentiable change of coordinates we can suppose that the inverse image of the $y$ axis is the $y$ axis itself. Let $L$ be the inverse image of the $y$ axis (that is to say the geodesic joining 0 to $\infty$ ). Then $L$ meets each horizontal hyperplane only once (these hyperplanes are the orbits of the action of the $\varphi^{*}\left(\operatorname{proj}_{X_{i}}\right)$ 's) and by hypothesis is a border-transversal $\mathcal{C}^{k}$ submanifold of the closed half space. Hence

$$
L=\left\{\left(f_{1}(y), \ldots, f_{n}(y), y\right) ; y \in \mathbb{R}^{+}\right\}
$$

where $f_{i}$ 's are $\mathcal{C}^{k}$ maps. The differentiable change of coordinates

$$
\left(x_{1}, \ldots, x_{n}, y\right) \longmapsto\left(x_{1}-f_{1}(y), \ldots, x_{n}-f_{n}(y), y\right)
$$


transforms $L$ into the $y$ axis and do not change $\varphi^{*}\left(\operatorname{proj}_{X_{i}}\right)$.

When the inverse image of the $y$ axis is the $y$ axis itself, there is a continuous $\operatorname{map} f: \mathbb{R}^{+} \longmapsto \mathbb{R}^{+}$such that

$$
\varphi(0, \ldots, 0, y)=(0, \ldots, 0, f(y)) .
$$

Since $\varphi^{*}\left(\frac{\partial}{\partial x_{i}}\right)=\frac{\partial}{\partial x_{i}}$ we have

$$
\varphi\left(x_{1}, \ldots, x_{n}, y\right)=\left(x_{1}, \ldots, x_{n}, f(y)\right) .
$$

There is a projective geodesic which may be written at 0 as

$$
\left\{\left(x, k_{2}(x), \ldots, k_{n}(x), k_{n+1}(x)\right) ; x \in[0, \varepsilon]\right\}
$$

where $k_{i}$ 's are $\mathcal{C}^{k}$ maps; let $L_{2}$ be its inverse image.

By hypothesis, we can write $L_{2}=\left\{\left(l_{1}(y), \ldots, l_{n}(y), y\right) ; y \in \mathbb{R}^{+}\right\}$where $l_{i}$ 's are $\mathcal{C}^{k}$ maps. Then computing $\varphi\left(L_{2}\right)=\left\{\left(l_{1}(y), \ldots, l_{n}(y), f(y)\right) ; y \in \mathbb{R}^{+}\right\}$gives $f=k_{n+1} \circ l_{1}$, hence $f$ is $\mathcal{C}^{k}$.

\subsection{Transversality of geodesics in dimension at least 3}

Lemma 6 Let $\varphi$ be a $\mathcal{C}^{k}$ compactification of isom. If $n \geqslant 3$ then the inverse images of the projective geodesics are $\mathcal{C}^{k}$ border-transversal submanifolds.

Proof: If $n$ is odd, each geodesic is the set of fixed points of a symmetry whose differentials in the endpoints of the geodesic has the form $\left(\begin{array}{cccc}1 & & & \\ & -1 & & \\ & & \ddots & \\ & & & -1\end{array}\right)$.

If $n$ is even, each geodesic is the set of common fixed points of two symmetries around totally geodesic planes whose differentials in the endpoints of the

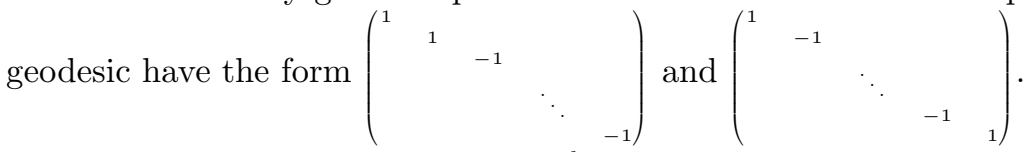

In both cases, we get implicit $\mathcal{C}^{k}$ definitions of the geodesics, which are therefore border-transversal $\mathcal{C}^{k}$ submanifolds of the closed ball.

With lemmas 5 and 6 we have proved that any compactification is given by a map $\varphi_{f}$ of the form (2) for some $\mathcal{C}^{k}$ map $f$.

It is clear that two such compactifications are conjugate if and only of the corresponding maps $f$ 's are.

\subsection{End of proof of Theorem 1}

Concerning Theorem 1 we are left with proving that we can replace the map $f$ by a monomial map and that any monomial map gives a compactification.

We omit the proof of the following classical fact.

Lemma 7 In the analytic case, we can replace $f$ by a map of the form $y \longmapsto y^{p}$ 
Lemma 8 Let $\bar{X}$ be an analytic vector field on the closed half-plane, tangent to the boundary. Then for any integer $p$, the pull-back of $\bar{X}$ by $\left(x_{1}, \ldots, x_{n-1}, y\right) \longmapsto$ $\left(x_{1}, \ldots, x_{n-1}, y^{p}\right)$ extends analytically to the boundary.

Proof: since $\bar{X}$ is analytic, it has the form

$$
\bar{X}=\sum_{i=1}^{n} \sum_{a, b} \alpha_{a, b}^{i} x^{a} y^{b} \frac{\partial}{\partial x_{i}}+\sum_{a, b} \beta_{a, b} x^{a} y^{b} \frac{\partial}{\partial y}
$$

where the sums are taken over all non-negative integers $b$ and all $n$-tuples of non-negative integers $a ; x^{a}$ means $x_{1}^{a_{1}} x_{2}^{a_{2}} \ldots x_{n}^{a_{n}}$.

We denote by $\varphi_{p}$ the map $\left(x_{1}, \ldots, x_{n-1}, y\right) \longmapsto\left(x_{1}, \ldots, x_{n-1}, y^{p}\right)$. After a direct computation, we see that

$$
\varphi_{p}^{*}(\bar{X})=\sum_{i=1}^{n} \sum_{a, b} \alpha_{a, b}^{i} x^{a} y^{p b} \frac{\partial}{\partial x_{i}}+\sum_{a, b} \beta_{a, b} x^{a} y^{p b+1-p} \frac{\partial}{\partial y}
$$

and hence, it is analytic if for all $a, \beta_{a, 0}=0$, that is to say if $\bar{X}$ is tangent to the boundary.

Corollary 9 Let $p$ be a positive integer. Then the map $\varphi_{p}$ given by (11) is a compactification.

Proof: Lemma 8 ensures that the pull-back of the action of the Lie algebra of $G$ admits an extension to the closed ball into an analytic action. This action is complete by compacity, thus it gives an action of the universal cover $\widetilde{G}$ of $G$. Let $g$ be an element $\widetilde{G}$ which projects on identity. Then $g$ acts trivially in the open ball, therefore it acts trivially in the whole closed ball. Thus, the action of $\mathfrak{g}$ gives an action of $G$.

\subsection{End of proof of Theorem 2}

Concerning Theorem 2 we are left with proving that the map $\varphi_{f}$ given by (2) is a compactification if and only if $f$ satisfies (3) and that this condition is satisfied by all non-flat maps.

Lemma 10 In any dimension $n \geqslant 2, \varphi_{f}$ is a smooth compactification if and only if $f$ satisfies (3)

Proof: As seen in the proof of 9 gives a compactification of isom if and only if the vector fields $\varphi^{*}\left(\operatorname{proj}_{X}\right)$ admits a $\mathcal{C}^{\infty}$ extension to the boundary.

Let $H$ be a hyperbolic element of $\mathfrak{g}$ stabilizing the $y$ axis, $\left(X_{i}\right)_{1 \leqslant i \leqslant n-1}$ be a basis of the vector space of the parabolic elements of $\mathfrak{g}$ stabilizing $\infty$ (the only point not contained in the chart $\mathcal{K C}),\left(Y_{i}\right)_{1 \leqslant i \leqslant n-1}$ be a basis of the vector space of the parabolic elements of $\mathfrak{g}$ stabilizing 0 , and $\left(R_{j}\right)_{1 \leqslant j \leqslant \frac{(n-1)(n-2)}{2}}$ be a basis of the vector space of the elliptic elements of $\mathfrak{g}$ stabilizing (pointwise) the $y$ axis. 
The union of this elements generates $\mathfrak{g}$, thus we only have to check that they all admit smooth extensions in the model given by $\varphi$.

The $\operatorname{proj}_{X_{i}}$ 's and $\operatorname{proj}_{R_{j}}$ 's are left unchanged by $\varphi$, thus admit extensions.

The $Y_{i}$ 's are conjugate one to another by rotations of $G$ generated by the $R_{j}$ 's, so we just have to check one of them.

We can explicitly compute $\widetilde{H}=\varphi^{*}\left(\operatorname{proj}_{H}\right)$ and one of the $\tilde{Y}_{i}=\varphi^{*}\left(\operatorname{proj}_{Y_{i}}\right)$. We first give the explicit expressions of $\operatorname{proj}_{H}$ and $\operatorname{proj}_{Y_{1}}$ in the chart $\mathcal{K C}$, computed thanks to (4).

$$
\begin{aligned}
& \operatorname{proj}_{H}\left(x_{1}, \ldots, x_{n-1}, y\right)=\quad \begin{array}{l}
2 x_{1} \\
2 x_{2} \\
\ldots \\
2 x_{n-1} \\
4 y
\end{array} \\
& \operatorname{proj}_{Y_{1}}\left(x_{1}, \ldots, x_{n-1}, y\right)=\mid \begin{array}{l}
y+x_{2}^{2}+x_{3}^{2}+\cdots+x_{n-1}^{2}-x_{1}^{2} \\
-2 x_{1} x_{2} \\
-2 x_{1} x_{3} \\
\ldots \\
-2 x_{1} x_{n-1} \\
-4 x_{1} y
\end{array} \\
& \widetilde{H}\left(x_{1}, \ldots, x_{n-1}, y\right)=\quad \begin{array}{l}
2 x_{1} \\
2 x_{2} \\
\ldots \\
2 x_{n-1} \\
4 f(y) / f^{\prime}(y)
\end{array} \\
& \tilde{Y}_{1}\left(x_{1}, \ldots, x_{n-1}, y\right)=\mid \begin{array}{l}
f(y)+x_{2}^{2}+x_{3}^{2}+\cdots+x_{n-1}^{2}-x_{1}^{2} \\
-2 x_{1} x_{2} \\
-2 x_{1} x_{3} \\
\ldots \\
-2 x_{1} x_{n-1} \\
-4 x_{1} f(y) / f^{\prime}(y)
\end{array}
\end{aligned}
$$

We see that $\widetilde{H}$ and $\widetilde{Y}_{1}$ admit smooth extensions if and only if $f / f^{\prime}$ does.

Lemma 11 Let $f$ be a smooth homeomorphism of $\mathbb{R}^{+}$. If $f$ is non-flat, then it satisfies (3).

We omit the proof, a simple verification.

\section{References}

[1] Benoît Kloeckner. On differentiable compactifications of the hyperbolic plane and algebraic actions of $\mathrm{SL}(2, \mathbb{R})$ on surfaces. Available at www . umpa. ens-lyon.fr/ bkloeckn/. 
[2] Yoshihiko Mitsumatsu. SL $(2 ; \mathbf{R})$-actions on surfaces. In Geometric study of foliations (Tokyo, 1993), pages 375-389. World Sci. Publishing, River Edge, NJ, 1994.

[3] C. R. Schneider. SL $(2, R)$ actions on surfaces. Amer. J. Math., 96:511-528, 1974.

[4] Dennis C. Stowe. Real analytic actions of $\mathrm{SL}(2, \mathbf{R})$ on a surface. Ergodic Theory Dynam. Systems, 3(3):447-499, 1983.

UMPA, ÉNS Lyon

46, allée d'Italie 69364 Lyon cedex 07

France

WwW . umpa . ens-lyon. fr/ bkloeckn/ bkloeckn@umpa.ens-lyon.fr 